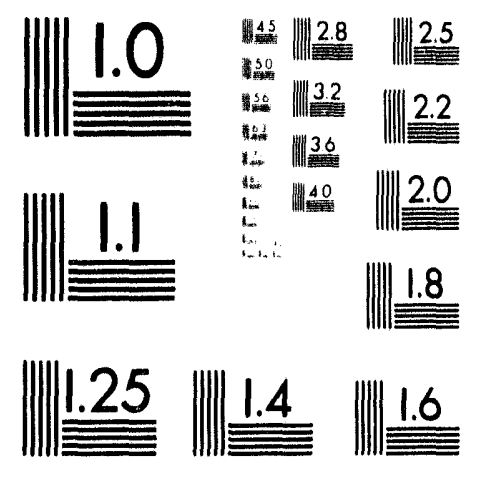



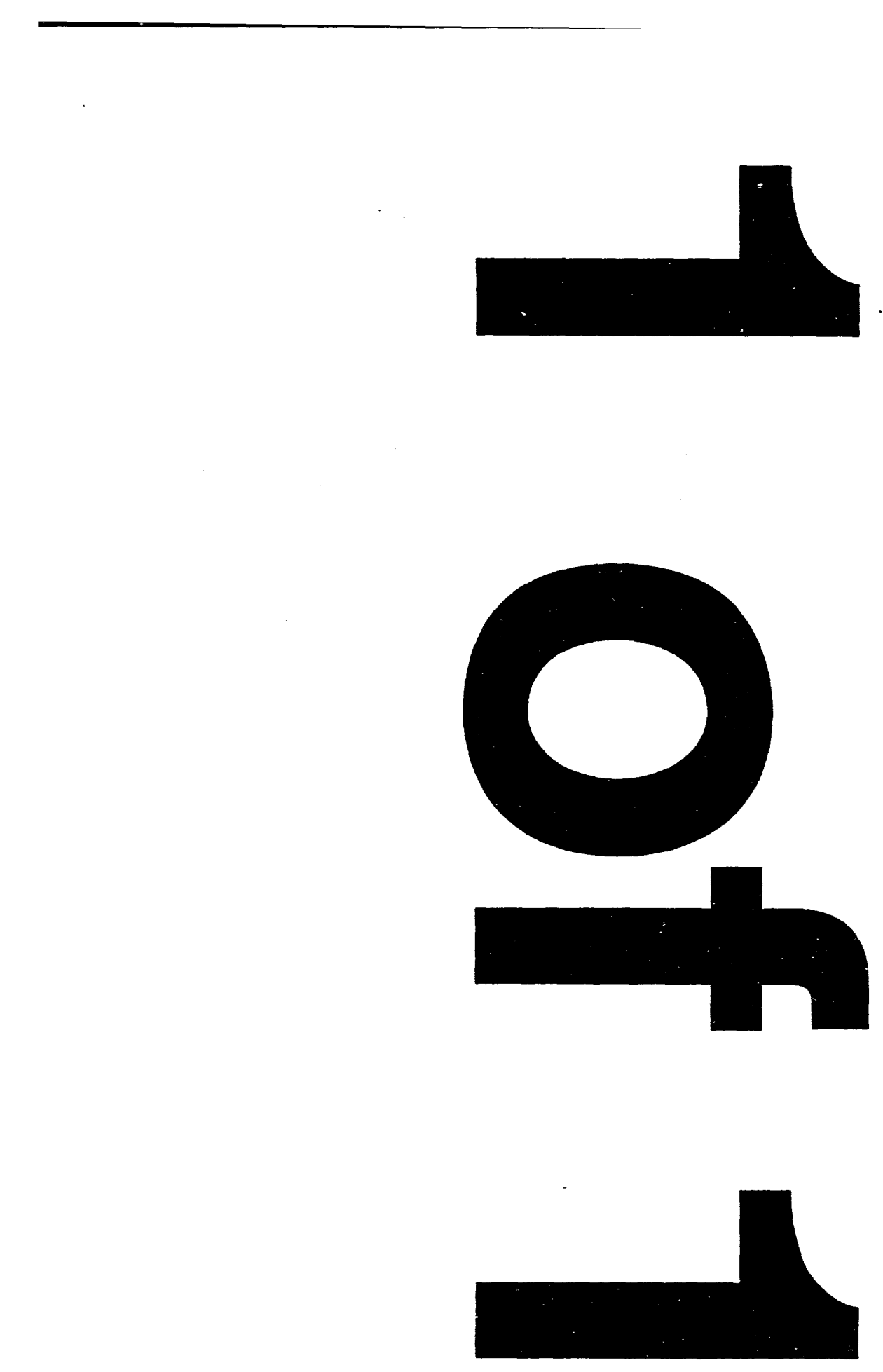


$$
\text { Conf } 930.8672=3
$$

\title{
Probing the nuclear multipole response with intermediate energy heavy-ion scattering
}

\author{
J. R. Beene
}

Oak Ridge National Laboratory, ${ }^{*}$ Oak Ridge, Tennessee, U.S.A.

The study of giant resonances using intermediate energy heavy-ion elastic scattering is discussed with emphasis on the potential of these probes for exploration of strengths above the giant dipole resonance (GDR) in singles and in decay experiments. Recent results on two phonon GDR strength are presented.

\section{INTRODUCTION}

The identification and characterization of the wide variety of collective modes that exist in the continuum region of the nuclear excitation spectrum continues to be a major goal of experimental nuclear structure physics. Recent results have demonstrated the considerable potential of intermediate energy heavy-ion inelastic scattering reactions as tools in these investigations [1-6]. Inelastic heavy ion scattering near $100 \mathrm{MeV} /$ nucleon excites giant multipole resonances (GR) with very large cross sections and very large resonance peak to underlying continuum ratios, and excite both isovector and isoscalar states with comparable cross sections. These features and others make intermediate energy heavy-ion scattering a useful tool for exploration of high-lying strength both in inelastic singles studies $[3,4]$, and especially in resonance decay studies $[1,2,6,7]$. The decay studies can be used both to increase specificity in strength distribution studies and to explore dynamics of resonance damping and decay processes themselves.

In this talk, I will explore a few areas in which I feel inelastic heavy ion scattering has particular promise as a tool for studying GR, and in which experimental results are available. The examples which I will cover depend on decay processes as tags for particular resonance modes, however I will not cover at all the study of the GR decay processes themselves and their relation to GR damping. Some of our most recent, most interesting (and most preliminary) results are in this area [6].

As an ad hoc definition of "intermediate energy," i.e. the energy range discussed in this talk, I will take $\sim 50$ to $200 \mathrm{MeV} /$ nucleon. Though the actual data I will discuss extends only to $95 \mathrm{MeV} /$ nucleon, the benefits of going to somewhat higher energy are considerable. The $200 \mathrm{MeV} /$ nucleon limit to the energy range is roughly defined by the experimental techniques we employ. We rely on high resolution magnetic spectrometers to provide a

\footnotetext{
* Managed by Martin Marietta Energy Systems, Inc. under contract DE-AC05-84OR21400 with the U.S. Department of Energy. 
direct determination of energy transfer and to serve as the primary trigger detector in our experiments. Current spectrometer designs achieve momentum resolution of $\Delta \mathrm{p} / \mathrm{p} \sim 10^{-4}$. GR studies require energy resolution comparable to the width of GR states -- i.e., 1 to 2 $\mathrm{MeV}$. This implies a limitation of beam energy to less than $\sim 10^{4} \mathrm{MeV}$.- or about 150 $\mathrm{MeV} /$ nucleon at mass 60 .

\section{EXPERIMENT}

The experiments reported here were studies of inelastic heavy ion scattering in coincidence with $\gamma$-rays and neutrons. All data were taken at GANIL using a high resolution magnetic spectrograph (SPEG) [8] for detection, identification and momentum analysis of inelastically scattered beam particles, and a large array of $\mathrm{BaF}_{2}$ detectors for identification and detection of decay products. Experiments have included scattering of $17 \mathrm{O},{ }^{36} \mathrm{Ar},{ }^{86} \mathrm{Kr}$, and ${ }^{64} \mathrm{Zn}$ from ${ }^{208} \mathrm{~Pb}$ and ${ }^{209} \mathrm{Bi}$ targets at $84,95,60$, and $80 \mathrm{MeV} /$ nucleon, respectively.

Space limitations preclude an adequate discussion of experimental details, or analysis technique here; only a bare outline will be provided. A somewhat more complete description of our most recent $\left(80 \mathrm{MeV} /\right.$ nucleon $\left.{ }^{64} \mathrm{Zn}\right)$ experiment is provided in $\mathrm{P}$. Mueller's contribution to this conference [6], see also refs. 1 and 5 for experimental information.

Different configurations for the $\mathrm{BaF}_{2}$ arrays have been used. For example, in the ${ }^{36} \mathrm{Ar}$ and ${ }^{86} \mathrm{Kr}$ bombardments $228 \mathrm{BaF}_{2}$ crystals arranged in 12 blocks of 19 detectors each were used. For details of this setup see ref. 5. Our most recent setup [2] employed the full (December 1992) implementation of the TAPS array [9] consisting of 5 blocks of 64 hexagonal crystals $(6 \mathrm{~cm} \times 25 \mathrm{~cm})$ each stacked in rectangular blocks centered on the reaction plane centered at angles of $\theta=51^{\circ}, 65^{\circ}, 101^{\circ}, 108^{\circ}$, and $147^{\circ}$ ranging from 60 to 65 $\mathrm{cm}$ from the target. Each of these 320 crystals subtended $\sim 6^{\circ}$, and was covered by a $5 \mathrm{~mm}$ thick plastic scintillator for charged particle identification and suppression [9]. An additional block of 19 crystals was positioned below the reaction plane at $\theta=90^{\circ}$. Another feature of this setup was a "forward wall" of plastic phoswitch detectors (see Mueller et al., ref. 6).

In all the systems studied, SPEG and its associated detector systems provided unit mass and $\mathrm{Z}$ identification of the ejectile and a momentum resolution of a few times $10^{-4}$.

\section{GIANT RESONANCE EXCITATION}

Both the nuclear and Coulomb interaction play a significant role in the excitation of GR by heavy ions. For bombarding energies below $\sim 50 \mathrm{MeV} /$ nucleon heavy ions are useful primarily as a tool for exploration of isoscalar strength. Above $50 \mathrm{MeV} /$ nucleon, as Coulomb excitation of high-lying strength becomes increasingly important, isovector strength can also be explored.

In general inelastic scattering of nuclear probes via the nuclear interaction favors isoscalar excitations because the isoscalar piece of the central nucleon-nucleon interaction is stronger than the isovector piece. For heavy ion scattering, insight into the relative excitation of isoscalar and isovector GR strength is best explored using the folding model $[10,11]$. One find that in general, if the nucleus being excited has $\mathrm{N} \neq \mathrm{Z}$, both the isoscalar and isovector pieces of the nucleon-nucleon interaction contribute to excitation of isovector states. For most heavy-ion probes (including those used here) the isoscalar interaction actually dominates the excitation of isovector states. Employing the folding model with simple assumptions concerning $n$ and $p$ densities (e.g., $\rho_{n}=N / A \rho$, and $\rho_{p}=Z / A \rho$ for all $r$ ), we can 
estimate that the transition potentials $\delta U_{1 t}$ for exciting collective $t=0$ and 1 states of the same $\ell$ are related hy $\delta U_{11} \approx[(\mathrm{N}-\mathrm{Z}) / \mathrm{A}] \delta \mathrm{U}_{10}$ for $\ell \geq 2$. Thus for isoscalar and isovector states with $\ell \geq 2$ at the same excitation energy having the same sum rule strength, the hadronic excitation cross section for the isovector state will be a factor of $[(\mathrm{N}-\mathrm{Z}) / \mathrm{A}]^{2}$ smaller $(\mathrm{N}, \mathrm{Z}, \mathrm{A}$ refer to the nucleus being excited) or about $1 / 20$ for $208 \mathrm{~Pb}$.

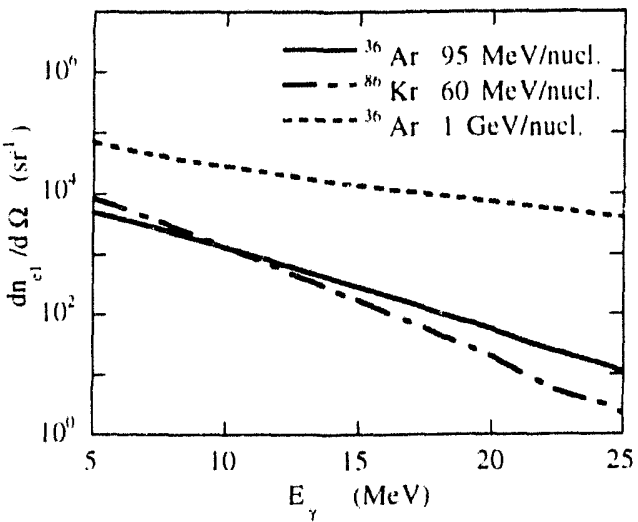

Figure 1. Virtual photon spectra seen by the target for the projectiles and bombarding energies indicated on the plot, corresponding, in each case, to the trajectory at the peak of the differential cross section.

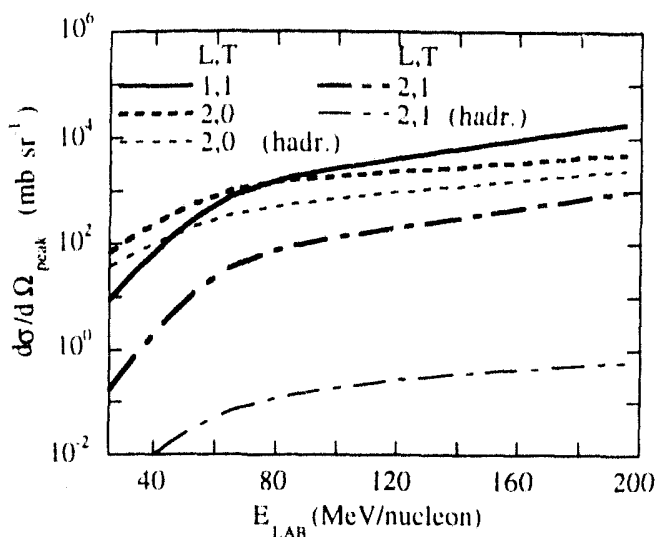

Figure 2. Peak differential cross sections for excitation of $\mathrm{GR}$ in $208 \mathrm{~Pb}$, labeled by angular momentum and isospin $(\mathrm{L}, \mathrm{T})$, as a function of bombarding energy for ${ }^{17} \mathrm{O}+{ }^{208} \mathrm{l}^{\prime} \mathrm{b}$.

For $\ell=1$ states the situation is more complex [11]. In the approximation considered for $\ell \geq 2$, the hadronic excitation for the isovector GDR vanishes. Some feeling for how small the nuclear contribution to GDR excitation is can be obtained from folding model calculations, with the interaction discussed in Section 3, the transition densities of ref. 11 , and using the isoscalar GQR for comparison. For ${ }^{36} \mathrm{Ar}$ scattering on ${ }^{208} \mathrm{~Pb}$ we find, at the strong absorption radius $(\mathrm{R} \sim 13.1 \mathrm{fm}), \delta \mathrm{U}_{11} / \delta \mathrm{U}_{20} \sim 1 / 100$.

Coulomb excitation is most familiar as a quantitative tool in study of low-lying collective states with beams at subbarrier energies. Giant resonances, however, lie at energies above $\sim 10 \mathrm{MeV}$. It is convenient to thirik of Coulomb excitation of high lying strength in terms of absorption of virtual photons -- the rapidly changing electromagnetic field as a projectile passes the target can be expressed in terms of a virtual photon field. Adequate, and informative calculations of Coulomb excitation amplitudes, and hence virtual photon spectra can be made using semiclassical techniques $[12,13]$. The excitation probability of a state at $E^{*}$ will be proportional to the intensity, $n_{\lambda}\left(E_{\gamma}\right)$, of the virtual photon field at $E_{\gamma}=E^{*}$ and the relevant photo-absorption cross sections at $E_{\gamma}$ In general the virtual photon spectrum in the $E_{\gamma} \geq 10 \mathrm{MeV}$ region has an exponential character with an intensity proportional to $Z^{2}$ and a slope which decreases with increasing beam velocity. This is illustrated in Fig. 1 which shows the El virtual photon spectra resulting from ${ }^{17} \mathrm{O},{ }^{36} \mathrm{Ar},{ }^{86} \mathrm{Kr}$. and ${ }^{60} \mathrm{Zr}$ bombardment of $208 \mathrm{~Pb}$ at $84,95,60$, and $80 \mathrm{MeV} /$ nucleon, as seen by the $\mathrm{Pb}$ nucleus. The higher the ion velocity the less virtual photon intensity depends on $E_{\gamma}$ (i.e. the flatter the spectrum). Even at $1 \mathrm{GeV} /$ nucleon, however, the variation of virtual photon intensity across the $\sim 4 \mathrm{MeV}$ width of the GDR in ${ }^{208} \mathrm{~Pb}$ is still substantial, as shown in Fig. 2, which compares $\mathrm{n}_{\mathrm{E}}\left(\mathrm{E}_{\gamma}\right)$ for 95 and $1000 \mathrm{MeV} /$ nucleon. The corresponding excitation cross section distributions for the GDR are shown. 
The increase in $E_{\gamma}>10 \mathrm{MeV}$ content of the virtual photon spectrum with increasing bombarding energy leads to a rapid increase in Coulomb excitation cross sections for GR states. Figure 3 shows the bombarding energy dependence of the peak differential cross section for excitation of the isospin 0 and 1 giant quadrupole resonances (GQR) in $208 \mathrm{~Pb}$ by ${ }^{17} \mathrm{O}$. Also shown are cross sections for hadronic excitation (Coulomb excitation set to zcro). The energies of the states are taken to be 10.6 and $22 \mathrm{MeV}$ (for $\mathrm{T}=0,1$ respectively), and each is assumed to exhaust its respective energy weighted sum rule fully. The cross section for excitation of the isovector GDR (at $13.5 \mathrm{MeV}$ ) is also shown in Fig. 3. Above $\sim 50$ $\mathrm{MeV} /$ nucleon, both isovector and isoscalar states (at least for small $f$ ) can be excited with very large cross sections because of the increasing effectiveness of Coulomb excitation.

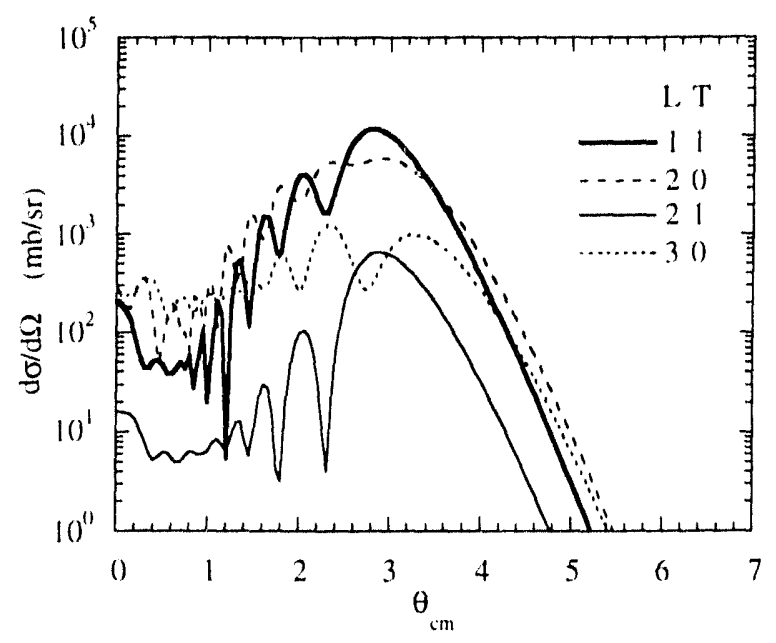

Figure 3. Cross sections for excitation of GR in of various $\mathrm{L}, \mathrm{T}$ in $208 \mathrm{~Pb}$ by $95 \mathrm{MeV} /$ nucleon ${ }^{36} \mathrm{Ar}$. $100 \%$ EWSR assumed for each mode.

Heavy ion inelastic scattering can excite GR states with much larger cross sections than proton and alpha scattering. However, this increase in cross section is bought at the price of almost complete loss of sensitivity of the inelastic scattering angular distribution to the angular momentum transfer. This is illustrated in Fig. 4. The angular distributions for excitation of isovector $\ell=1$ and $\ell=2$ states (almost purely Coulomb excited) are essentially identical; also shown is the angular distribution the isoscalar $\ell=2$ state (which has a significant hadronic component). While the angular distributions are clearly insensitive to l, they do carry information about the excitation mode (Coulomb, hadronic or mixed) of the state.

For states with a significant isoscalar component the nuclear and Coulomb cross sections can be comparable at angles near the grazing angle. This leads to a very favorable situation in which the isospin character (i.e., the ratio $M_{n} / M_{p}[11]$ ) can be accurately determined in a single measurement $[1,3,4,14]$ using Coulomb-nuclear interference. Such measurements are of critical importance, particularly for the GQR, since controversy persists (due largely to recent pion scattering measurements $[15,16]$ ) about $M_{n} / M_{p}$ of the "isoscalar" $G Q R$. If the pion data are correct, much of our current understanding of the isospin character of collective excitations must be revised.

\section{RESULTS FROM SINGLES SPECTRA}

\subsection{Elastic scattering and low-lying excited states}

Satchler $[17,3]$ has shown that elastic scattering of 17,160 from a variety of targets from a bombarding energy of $22 \mathrm{MeV} /$ nucleon to $84 \mathrm{MeV} /$ nucleon (including our data on $208 \mathrm{~Pb}$ ) can be described by a simpls double-folding model using an effective nucleon-nucleon interaction of Yukawa form with range $\lambda \sim 0.7 \mathrm{fm}$ and real and imaginary strength 
parameters given respectively by $u \approx w=54-0.22 \varepsilon \mathrm{MeV}$, where $\varepsilon$ is the bombarding energy in $\mathrm{MeV} /$ nucleon.

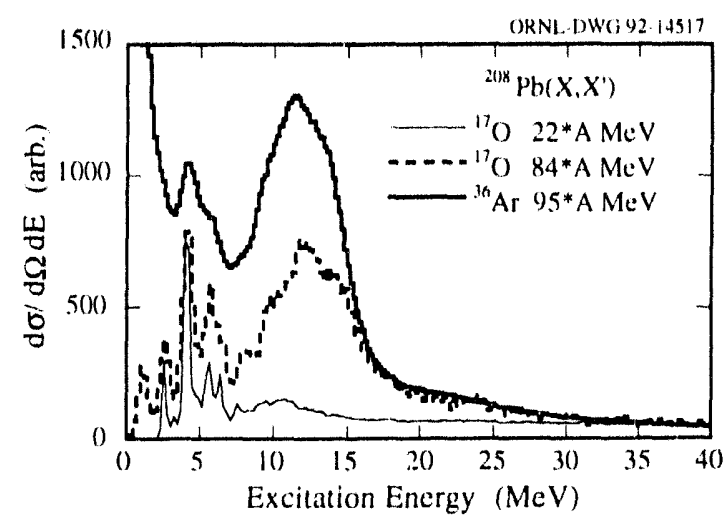

Figure 4. Inelastic singles spectra for scattering of $17 \mathrm{O}$ on $208 \mathrm{~Pb}$ at 22 and 84 $\mathrm{MeV} /$ nucleon and $36 \mathrm{Ar}+208 \mathrm{~Pb}$ at 95 $\mathrm{MeV} /$ nucleon. The spectra are normalized to be equal at $\mathrm{E}^{*}=35 \mathrm{MeV}$. distributions to the low-lying $2+(4.08 \mathrm{MeV})$ and $3^{-}(2.63 \mathrm{MeV})$ states of the target were also anaiyzed. We use the folding model, and a collective model description of the ${ }^{208} \mathrm{~Pb}$ transition densities. The results for both states were found to be consistent, within uncertainties $( \pm 15 \%)$ with the results of previous work (see ref. 1 and references therein). We stress the importance of using the folding model explicitly for calculation of transition potentials to be used in analysis of inelastic scattering data, especially for $\ell>2$ and for heavy ions. Thus one of the important aspect of the broad-ranged success of Satchler's simple Yukawa interaction above is that it could make routine use of the folding model in such analyses practical.

\subsection{Inelastic scattering in the GR region}

One of the most dramatic results [1,5] of our investigations of GR with heavy-ion inelastic scattering has been the very large improvement of peak to continuum ratios in the GR region with increasing E/A and $\mathrm{Z}$ of the projectile. This is illustrated in Fig. 5 which shows the inelastic spectra for ${ }^{208} \mathrm{~Pb}\left({ }^{17} \mathrm{O},{ }^{17} \mathrm{O}^{\prime}\right)$ at $22 \mathrm{MeV} /$ nucleon and $84 \mathrm{MeV} /$ nucleon, normalized to match at an excitation energy of $35 \mathrm{MeV}$. In Fig. 6 the data in Fig. 5 is reproduced with the addition of data for ${ }^{208} \mathrm{~Pb}\left({ }^{36} \mathrm{Ar},{ }^{36} \mathrm{Ar}\right)$ at $95 \mathrm{MeV} /$ nucleon. In proceeding from $22 \mathrm{MeV} /$ nucleon ${ }^{17} \mathrm{O}$ to $95 \mathrm{MeV} /$ nucleon ${ }^{36} \mathrm{Ar}$, the cross section in the resonance region has increased by a factor of almost 300 while the continuum cross section (at $E^{*}=35 \mathrm{MeV}$ ) has increased by a factor of less than 8 . These spectra are all for scattering angles corresponding to the peak of the GDR cross section; for reference the integrated cross section in the large peak at $12 \mathrm{MeV}$ for $95 \mathrm{MeV} /$ nucleon ${ }^{36} \mathrm{Ar}$ is $\sim 16$ barns/sr (E1 + E2), while the $35 \mathrm{MeV}$ continuum cross section is $\sim 120 \mathrm{mb}(\mathrm{MeV} \cdot \mathrm{sr})^{-1}$.

The very large cross sections for excitation of E1 and E2 strength in the GR region with $\sim 100 \mathrm{MeV} /$ nucleon heavy ions, and the very good peak to continuum ratio obviously suggests that such reactions are ideal for GR decay studies involving comparatively weak 
decay processes (e.g., $\gamma$-decays, semi-direct particle decays, etc.). See for example refs. [1], [2], [18], and the presentations of Mueller [6] and Van der Woude [7] at this conference.

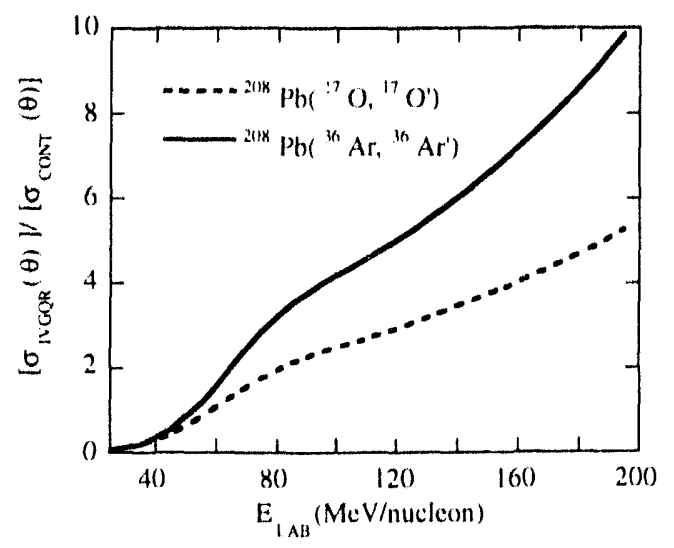

Figure 5. IVGQR resonance cross section in $208 \mathrm{~Pb}$ divided by the estimated continuum cross section for $\mathrm{Ar}+\mathrm{Pb}$ and $\mathrm{O}+\mathrm{Pb}$. The continuum $\sigma$ is in $\mathrm{mb} / \mathrm{MeV}$, while the GQR $\sigma$ is integrated over the $-5 \mathrm{MeV}$ wide peak. Thus a $1-$ to- 1 peak to continuum ratio is achieved when the ratio plotted here is $\sim 5$.

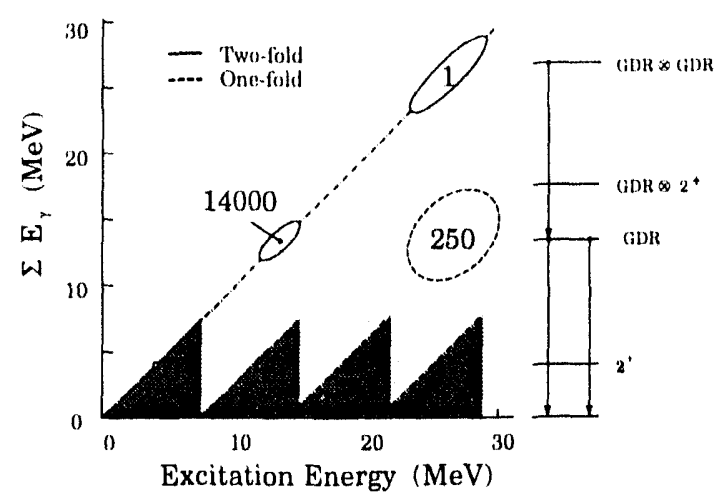

Figure 6. Schematic illustration of the experimental identification of the DGDR excitation by single $\gamma$-ray coincidence in the ${ }^{208} \mathrm{~Pb}\left(X, X^{\prime} \gamma\right)$ reaction. can be considered a harmonic vibration, there should be a finite probability for multiple excitation of the GDR mode (or for excitation of the GDR together with other collective modes). The spectroscopic properties of the two (or more) phonon GDR excitations are important since they should provide direct information about the harmonicity of the GDR mode and also potentially interesting information about decay processes and resonance damping. continuum excitation from a variety of heavy ion data including that in Fig. 4 was used to make an estimate of the energy and probe dependence of the continuum excitation. These systematics were used to construct the peak to continuum ratio plots for the isovector GQR in Fig. 5 (assuming $\mathrm{E}^{*}=22 \mathrm{MeV}$ and a strength of $50 \%$ of the isovector $1=2$ energy weighted sum rule). These results imply that at energies around $150 \mathrm{MeV} /$ nucleon, sensitive and systematic studies of the isovector GQR will be possible in heavy-ion singles. Our 95 $\mathrm{MeV} /$ nucleon $86 \mathrm{Ar}+208 \mathrm{~Pb}$ is much less favorable in peak-to-continuum ratio at $\mathrm{E}^{*}$ $25 \mathrm{MeV}$ than $150 \mathrm{MeV} /$ nucleon data would be, but useful results can be obtained. Singles analysis of the region above the GDR (16-30 $\mathrm{MeV})$, is consistent with localized $\ell=3$ strength ( $30 \%$ of EWSR) at $18 \mathrm{MeV}$ and $\sim 50$ $70 \%$ of the IVGQR EWSR centered at 22 $\mathrm{MeV}$. We have also searched for evidence of isoscalar dipole strength in this excitation energy region. Our preliminary conclusion is that our data are not consistent with previous proton and alpha scattering data suggesting a localized isoscalar dipole peak [18].

\section{TWO PHONON STRENGTH}

The most important mechanisms for excitation of GR by heavy ions were discussed in Section 3. Coulomb excitation dominates the GDR cross section for heavy ion beams above $~ 50 \mathrm{MeV} /$ nucleon. The Coulomb excitation probability for the GDR increases rapidly with increasing bombarding energy per nucleon and increasing $\mathrm{Z}$ of the projectile (for target excitation). To the extent that the GDR

A systematic investigation of the 
The general trends in excitation probability discussed above might lead one to assume that multiphonon strength is best studied with the highest energy heams available. This is not necessarily the case. In ${ }^{208} \mathrm{~Pb}$ the peak of the photo-absorption cross section to the GDR is at 13.5 MeV. The two phonon excitation process corresponds to the absorption of two $\sim 13$ $\mathrm{MeV}$ photons. At very high energies the virtual photon spectrum becomes sufficiently flat that absorption of a single $\sim 27 \mathrm{MeV}$ photon via $\ell>1$ multipole strength or the "quasideuteron" tail of the $\ell=1$ strength overwhelms the two photon cross section. In fact the optimum bombarding energy for studies of the two phonon GDR without use of a statespecific experimental tag turns out to be in the $500 \mathrm{MeV} /$ nucleon to few $\mathrm{GeV} /$ nucleon range. The first published data on the Coulomb excitation and direct identification of the DGDR comes from experiments at GSI in this bombarding energy range [20,21]. (The first experimental DGDR came from pion double charge exchange [22].)

Total angle integrated cross sections for excitation of the DGDR in ${ }^{208} \mathrm{~Pb}$ and ${ }^{209} \mathrm{Bi}$ using the comparatively low energy beams (60 to $95 \mathrm{MeV} /$ nucleon) are calculated to be in the best case a factor of $\sim 300$ times smaller than the cross section measured for the ${ }^{209} \mathrm{Bi}+{ }^{208} \mathrm{~Pb}$ reactions at $1 \mathrm{GeV} /$ nucleon [21]. Nevertheless, at the peak of the differential cross section -where we acquired data -- differential cross sections as large as hundreds of mb/sr are expected, roughly comparable to the cross section per $\mathrm{MeV}$ in the continuum at $27 \mathrm{MeV}$. If a tool sensitive enough to pick the DGDR out of the continuum can be employed, we can use the advantages of direct, high resolution, determination of the excitation energy provided by the magnetic spectrometer to study the DGDR. There are two further features of the low energy data which make some compensation for the small cross section. The range of impact parameters to be included in the semiclassical calculation of DGDR excitation is a source of uncertainty [23]. We measure differential cross sections over a small range of angles near the grazing angle. Thus, in contrast to measurements which sum over angle, our range of impact parameters is well defined. In fact, the inelastic scattering angular distribution for the single phonon excitation provides experimental measure of both the impact paraneter distribution and the nuclear absorption as a function of impact parameters. A second feature of the low energy data is that the excitation probabilities remain fairly small. On the Coulomb trajectory corresponding to the peak of the two phonon cross sections, excitation probabilities reach only a few percent. In contrast for the GSI experiments [20,21], excitation probabilities exceed $30 \%$ - leading to the risk that the simple models used to describe the excitations might be inadequate.

The sensitive tool which we employ to identify the DGDR is photon decay. The photon decay of the collective GDR phonon provides an extremely strong E1 $\gamma$-ray transition which competes favorably with the dominant neutron decay and overwhelms other electromagnetic multipoles. In our previous work [19], we have illustrated how ground state photon decay can be used to isolate the one phonon GDR, even when it is buried in an overwhelming background. The use of $\gamma$-ray decay in concert with excitation energy measurement via magnetic spectrometer is illustrated in Fig. 7. An ideal situation would correspond to the detection of two $\sim 13.5 \mathrm{MeV} \gamma$-rays in coincidence de-exciting the two GDR phonons, in coincidence with scattered projectiles with momenta corresponding to $\sim 27 \mathrm{MeV}$ excitation energy. The region where such two-fold $\gamma$-ray events would appear on Fig. 7 is indicated by the oval lying along the $E^{*}=\Sigma E_{\gamma}$ line, near $E^{*}=27 \mathrm{MeV}$. Unfortunately, the DGDR excitation cross section coupled with the GDR $\gamma$-decay branching ratio and the solid angle covered by our $\gamma$-detector array make the yield these events impossibly small. Also indicated on the plot are the position at which events in which only a single GDR decay $\gamma$-ray $\left(E_{\gamma} \sim 13\right.$ $\mathrm{MeV}$ ) is detected. For our setup, this yield, for $208 \mathrm{~Pb}$ should be $\sim 250$ times larger than the yield of the $\gamma-\gamma$ events. This, coupled with excitation energy measurement, gives a very 
sensitive tag for the DGDR, since background due statistical emission of $\sim 13 \mathrm{MeV} \gamma$-rays in this excitation energy is calculated to be more than an order of magnitude smaller.

In Fig. 7, data acquired using this identification method are shown for three reactions, as indicated in the figure caption. The comparison of data from different probes is an important aspect of this work, since at the same projectile velocity, the DGDR cross
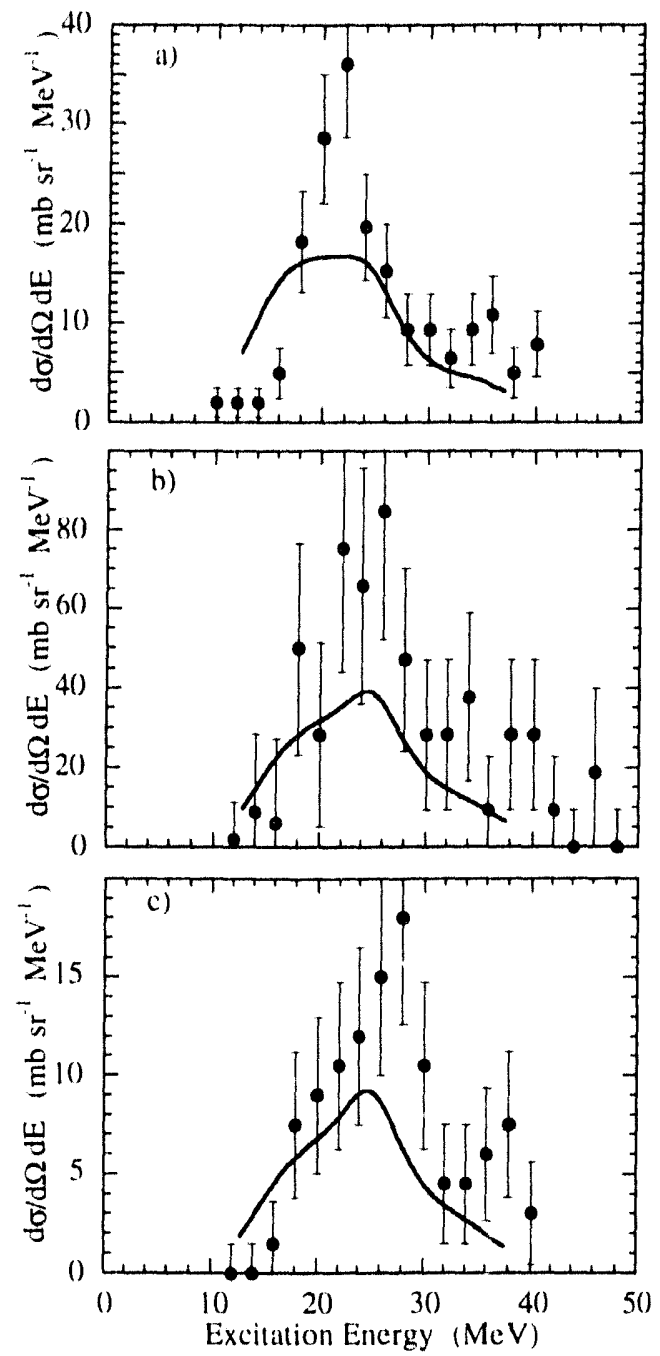

Figure 7. Experimental (points) and theoretical (curves) cross sections for DGDR excitation in target nuclei: a) $60 * \mathrm{~A} \mathrm{MeV}{ }^{86} \mathrm{Kr}$ on ${ }^{208} \mathrm{~Pb}$, b) $80^{*} \mathrm{~A} \mathrm{MeV}{ }^{64} \mathrm{Zn}$ on ${ }^{209} \mathrm{Bi}$, c) $95^{*} \mathrm{~A} \mathrm{MeV}{ }^{36} \mathrm{Ar}$ on $208 \mathrm{~Pb}$.

where the $Y$ 's are $\gamma$-ray yields, the $\sigma$ 's are cross sections, and the $R_{\gamma}$ are branching ratios for the DGDR to GDR $\gamma$-ray transition $(2 \rightarrow 1)$ and GDR to ground transition $(1 \rightarrow 0)$, respectively. If we assume $R_{\gamma}(2 \rightarrow 1) \approx R_{\gamma}(1 \rightarrow 0)$ (a plausible assumption; see $[5,21]$ ), then the $\sigma_{D G D R}$ can be calculated from the yield ratios and the calculated cross section for exciting the GDR $\left(E^{*}>9 \mathrm{MeV}\right)$. 


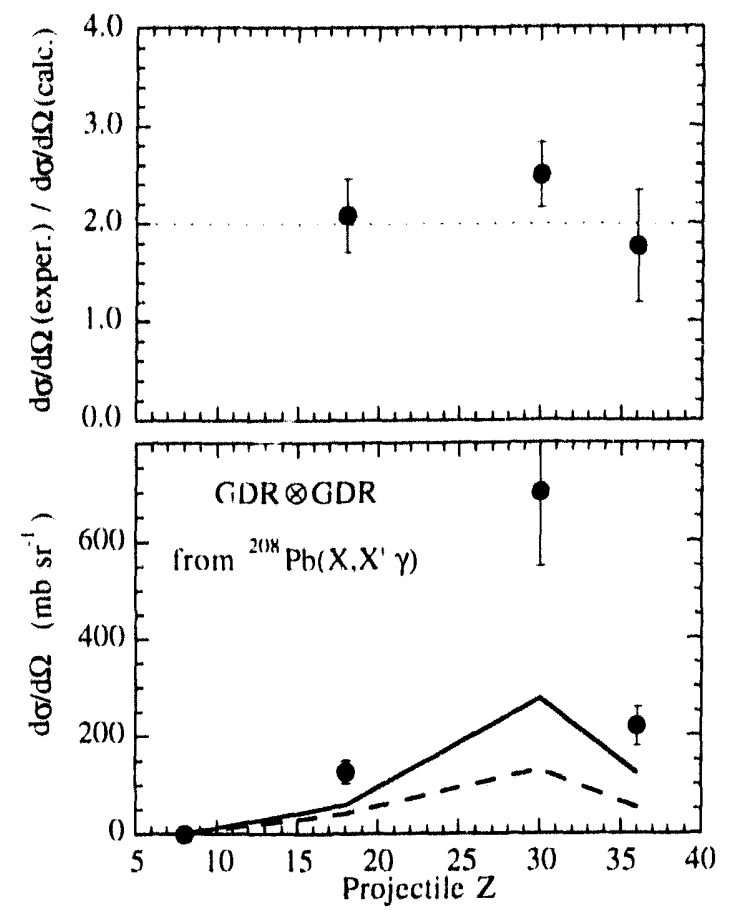

Figure 8. Cross sections for DGDR excitation. On the lower panel the energy-integrated cross sections from the data in Fig. 8 are plotted as a function of projectile $Z$. The solid line corresponds to the theoretical curves in Figure 7. The dashed line shows the cross section calculated for a sharp-line GDR (i.e. ignores peak width). The upper panel is the ratio of experimental data to theory. The choice of $\mathrm{Z}$ for an abscissa is for convenience. Since different projectile velocities are involved, the variation in $\sigma$ with $Z$ is not simply a $Z$ effect. effect is still significant at $1 \mathrm{GeV} /$ nucleon though it is much larger at the lower bombarding energies. The effect of variation of the $\gamma$-decay branching ratio across the resonance must also be considered in interpreting both our data and that of Ref. 21.

\section{SUMMARY}

We have discussed results on elastic and inelastic scattering of intermediate energy ${ }^{17} \mathrm{O}$, ${ }^{36} \mathrm{Ar},{ }^{36} \mathrm{Kr}$, and ${ }^{64} \mathrm{Zn}$ concentrating primarily on the excitation of the GR region. Preliminary results suggest that elastic scattering and inelastic excitation of low-lying states is well described by a folding with a simple Yukawa interaction introduced by Satchler [17]. Inelastic scattering in the giant resonance region is dominated by the isoscalar GQR and the 
isovector GDR. A multipole decomposition of the region just above GDR was undertaken, which is consistent isoscalar with E.3 strength at $18 \mathrm{McV}$ and isovector E2 strength $(\sim 50) \%$ EWSR) at $22 \mathrm{MeV}$. No evidence for isoscalar 1 - excitation in this region is found.

An effort was made to identify the DGDR in ${ }^{208} \mathrm{~Pb}$ and ${ }^{209} \mathrm{Bi}$ by a $\gamma$-deciay coincidence technique. We find cross section distributions consistent with a DGDR strength distribution implied by the convolution picture of ref. 24. The yield of photons which we identify with the decay of the DGDR is a factor of -2 larger than calculated from semiclassical cross sections and reasonable assumptions about the $\gamma$-decay branching ratio of the DGDR. Our results could imply either a larger than expected $\gamma$-branch $R(2 \rightarrow 1)$, or an enhancement of the DGDR excitation process over semiclassical expectations; or a combination of the two. While the first of these possibilities might seem the most likely, it should be pointed out that this factor of $\sim 2$ enhancement of DGDR cross section is a common feature of all Coulomb excitation studies reported so far $[20,21,25]$. The data from the ${ }^{64} \mathrm{Zn}$ (preliminary analysis) and ${ }^{86} \mathrm{Kr}$ bombardments are consistent with a DGDR strength at $2 * \mathrm{E}_{\mathrm{GDR}}=27 \mathrm{MeV}$ within an uncertainty of $\sim 0.6 \mathrm{MeV}$. The ${ }^{36} \mathrm{Ar}$ data give $\mathrm{E}_{\mathrm{DGDR}} \sim 28.5 \pm 1.0 \mathrm{MeV}$. It is net yet clear how well our data determine the width of the underlying strength function; this width cannot be read directly from the cross section distributions, but must be deduced from comparison with calculations. Preliminary results using all three data sets together suggest a width for the DGDR strength about 20\% smaller than predicted by the convolution calculations. Our calculations use a standard Lorentzian-peak parameterization of the GDR strength, and therefore imply $\Gamma_{D G D R} \sim 2 * \Gamma_{\text {GDR }}$. Our analysis gives $\Gamma_{D G D R} \sim 1.6 * \Gamma_{\text {GDR }}$ with an uncertainty in the factor of about $15 \%$. This is roughly consistent with results of higher energy Coulomb excitation work $[20,21]$.

This report is too short to allow discussion of many important aspects of these experiments -- both experimental difficulties and physics results. Some of these are discussed by Mueller [6]. The work discussed in this report has been done in close collaboration with my colleagues at ORNL including F. Bertrand, D. Horen, P. Mueller, R. Varner, M. Halbert, and D. Olive. Collaborators from other institutions have also contributed significantly. Those involved in recent work include Y. Schutz, W. Mittig, P. RousselChomaz, P. Laqutridou, F. Lefevre, M. Marqués, T. Matulewicz, and R. Ostendorf of GANIL; J. van Pol, and H. W. Wilschut of KVI; J. L. Ferrero and A. Marín of Valencia; R. Holtzmann of GSI; and M. Thoennessen and B. Sherrill of MSU. The most recent experiment benefited from the work of the entire TAPS collaboration, including members from GSI and Giessen not directly involved in our experiments. The design and implementation of the "forward wall" hodoscope was the work of the KVI group, as part of the TAPS collaboration.

\section{REFERENCES}

1. J.R. Beene, F.E. Bertrand, D.J. Horen, R.L. Auble, B.L. Burks, J. Gomez del Campo, M.L. Halbert, R.O. Sayer, W. Mittig, Y. Schutz, J. Barrette, N. Alamanos, F. Auger, B. Fernandez, A. Gillibert, B. Haas, and J.P. Vivien, Phys. Rev. C41 (1990) 920, and J. Barrette, N. Alamanos, F. Auger, B. Fernandez, A. Gillibert, D.J. Horen, J.R. Beene, F.E. Bertrand, R.L. Auble, B.L. Burks, J. Gomez del Campo, M.L. Halbert, R.O. Sayer, W. Mittig, Y. Schutz, B. Haas, and J.P. Vivien, Phys. Lett. B209 (1988) 182.

2. J.R. Beene, F.E. Bertrand, D.J. Horen, J.L. Lisantti, M.L. Halbert, D.C. Hensley, W. Mittig, Y. Schutz, J. Barrette, N. Alamanos, F. Auger, B. Fernandez, A. Gillibert, B. Haas, J.P. Vivien, and A.M. Nathan, Phys. Rev. C41 (1990) R13.32. 
3. D.J. Horen, R.L. Auble, J. Gumez del Campo, G.R. Sulchler, R.L. Varner, J.R. Beene. Brian Lund, V.R. Brown, P.L. Anlhony, and V.A. Madsen, Pliys. Rev. (47 (140)11 (2))

4. D.J. Horen, F.E. Bertrand, J.R. Beene, G.R. Salchler, W. Millig. A.C.C. Villari, Y. Schutz, Zhen Weniong, E. Plagnol, and A. Gillibert, Phys. Rev, (4 $4(190) 112.385$ and D.J. Horen, R.L. Auble, J.R. Beene, F.E. Bertrand, M.L. Hallhert, G.R. Salteliler. M. Thoennessen, R.L. Varner, V.R. Brown, P.L. Anthony, and V.A. Madsen, Pliys. Rev. C44 (1991) 128.

5. J.R. Beene and F. E. Bertrand, Nucl. Phys, A538 (1992) 55.30.

6. P.E. Mucller et al., these proceedings.

7. A. Van der Woude et al., these proceedings.

8. L. Bianchi, B. Fernandez, J. Gastebois, A. Gillibert, W. Millig. and J. Barrette, Phys. Rescurch A276 (1989) 509.

9. R. Novolny, IEEE Transiactions on Nuclear Science, 38 (1991) 379.

10. G.R. Satchler, Direct Nuclear Reilctions (Oxford University Press, New York. 198.3).

11. G.R. Salchler, Nucl. Phys. A472 (1987) 215.

12. A. Winther and K. Alder. Nucl. Phys. A319 (1979) 518.

13. C.A. Bertulani and G. Baur, Phys, Reports 163 (1088) 290.

14. G.R. Satchler, Nucl. Phys A491(1989)413.

15. S.J. Seestrom-Morris, C.L. Morris, J.M. Moss, T.A. Carey, D. Drake, J. C. Dousne, L.C. Bland, and G.S. Adams, Phys. Rev. C33 (1986) 1847.

16. J.L. Ullmann, P.W.F. Alons, B.L. Clausen, J.J. Kraushaar, J.H. Mitchell, R.J. Pelersum. R.A. Ristinen, R.L. Boudrie, N.S.P. King, C.L. Morris, J.N. Knudson, and E.F. Gibson, Phys. Rev. C35 (1987) 1099.

17. G.R. Satchler, unpublished (see also refi. 3).

18. G. S. Adams, T.A. Carey, J.B. McClelland, J.M. Moss, S.J. Secstrom-Morris, and D. Cook Phys, Rev C33 (1986) 2054, and references lherein.

19. J.R. Beene, F.E. Bertrand, M.L. Halbert, R.L. Auble, D.C. Hensley, D.J. Horen, R.I.. Robinson, R.O. Sayer and T.P Sjoreen, Phys. Rev. C39 (1989) 1.3(17.

20. R. Schmidt, Th. Blaich, Th. W. Elze, H. Emling, H. Freiesleben, K. Grimm, W, Henning, R. Holzmann, J.G. Keller, H. Klingler, R. Kulessu, J.V. Kratz, D. Lambrechl. J.S. Lange, Y. Leifels, E. Lubkiewicz, E.F. Moore, E. Wajda, W. Prokopowič Ch. Schütter, H. Spies, K. Stelzer, J. Stroth, W. Walus, H.J. Wollersheim, M. Zinser, and E. Zude, Phys. Rev. Lett. 70 (1993) 1767.

21. J. Ritman, F.-D. Berg, W. Kühn, V. Metig, R. Novotny, M. Notheisen, P. Paul, M. Pfeiffer, O. Schwalb, H. Löhner, L. Venema, A. Gobbi, N. Herrmann, K.D. Hildenbrand, J. Mösner, R.S. Simon, K. Teh, J.P. Wessels, and T. Wienuld, Phys. Rev. Lett. 70 (1993) 533.

22. S. Mordechai, N. Auerbach, M. Burlein, H.T. Fortune, S.J. Grecne, C. Fred Monre, C.L. Morris, J.M. O'Donnell, M.W. Rawool, J.D. Silk, D.L. Watson, S.H. Yoo, and J.D. Zumbro, Phys. Rev. Lett. 61, (1988) 531.

23. C.A. Bertulani and V. Zelevinski, Phys. Rev. Letl. 71 (1993) 967.

24. W.J. Llope and P. Braun-Munzinger, Phys. Rev. C41 (1990) 2644.

25. T. Aumann, J.V. Kratz, E. Stiel, K. Summerer, W. Brilchle, M. Schiidel, G. Wirth, M. Fauerbach, and J. C. Hill, Phys. Rev. C47 (1993) 1728.

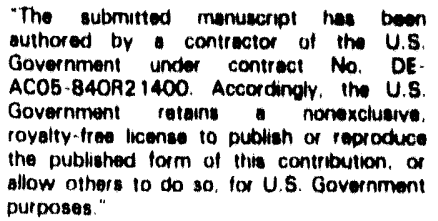



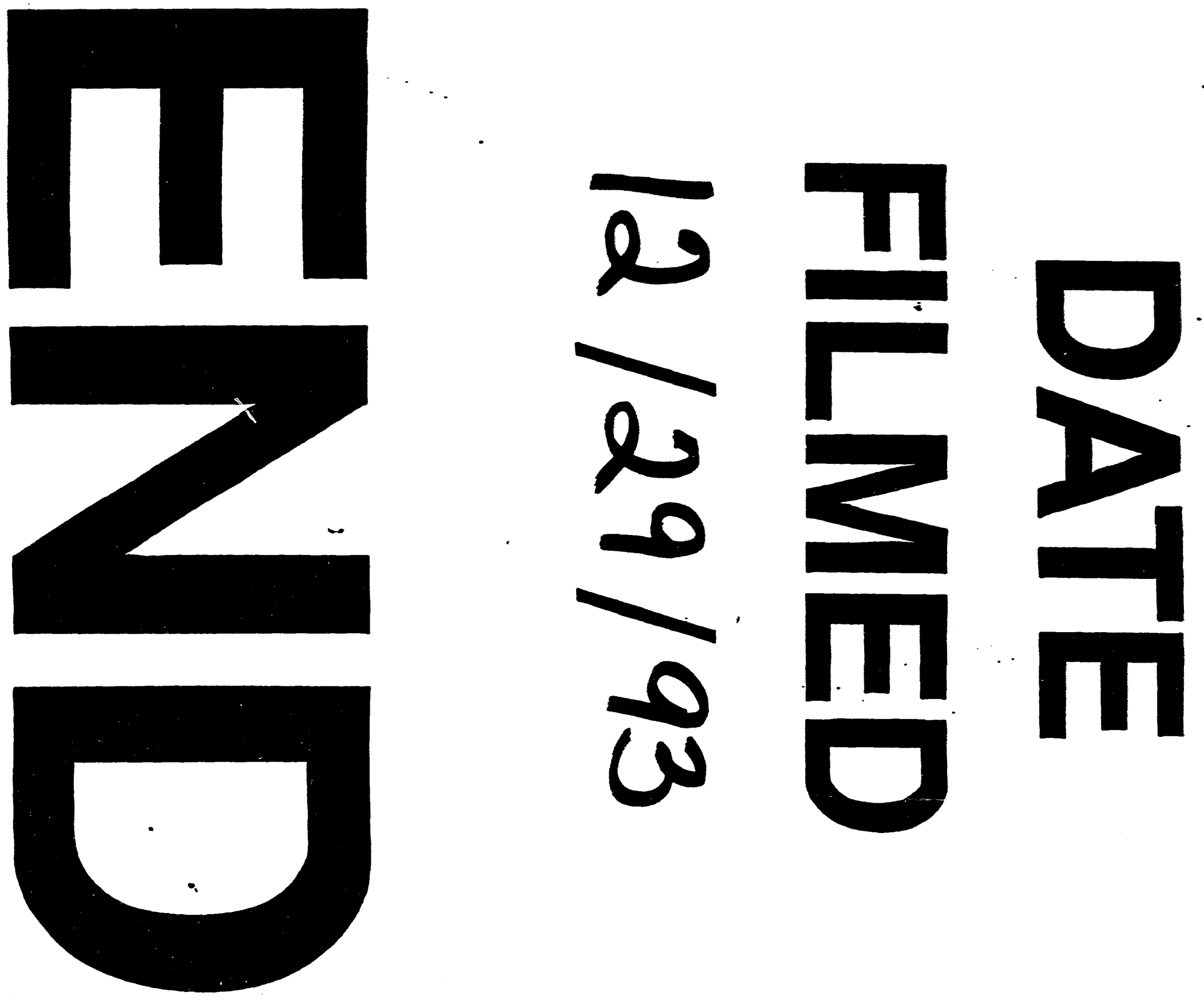
- 